\title{
Electron transfer photochromism of Ln-based (Ln = Dy, Tb) coordinated polymers for reversibly switching off/on single-molecule magnetic behavior
}

\author{
Qian Zhang ${ }^{\dagger}$, Song-De $\mathrm{Han}^{\dagger}$, Qi Li, Ji-Xiang $\mathrm{Hu}^{*}$ and Guo-Ming Wang*
}

\begin{abstract}
Switching on/off single-molecule magnets (SMMs) at room temperature is still a challenge in moleculebased magnets. Herein, two photochromic Ln-based ( $\mathrm{Ln}=\mathrm{Dy}$, $\mathrm{Tb})$ phosphonate coordinated polymers were synthesized with regulable SMM behavior. The reversible room-temperature photo-coloration was an electron transfer process with a generation of relatively stable radicals, characterized by structural analyses, ultraviolet-visible, luminescence and electron spin resonance spectra and magnetic measurements. Importantly, owing to the antiferromagnetic coupling interactions between $\mathrm{Ln}^{3+}$ ions and photogenerated radicals, the room-temperature light irradiation surprisingly switched off the SMM behavior, showing the first example of radicalquenched SMMs in the molecule-based magnets. Moreover, the silient SMM behavior could be recovered after eliminating photogenerated radicals via heat treatment, showing a reversible off/on switch of SMMs via light and heat. This work constructs a system for switching off/on SMMs through electron transfer photochromism, providing a visual operation way via naked-eye-detectable coloration for the switchable SMMs.
\end{abstract}

Keywords: electron transfer, photochromism, photogenerated radicals, single-molecule magnets, switch off

\section{INTRODUCTION}

Single-molecule magnets (SMMs), exhibiting slow magnetic relaxation resulting from the bistable magnetic ground states, can serve as attractive candidates for next generation highdensity information storage devices [1-3]. Since the discovery of the well-known nanoscale $\mathrm{Mn}_{12}$ molecule, many efforts have been devoted to exploring excellent SMMs based on transition metals. More recently, in pursuing the preparation and construction of SMMs with large effective energy barriers and blocking temperatures, lanthanide (Ln)-containing complexes soon stand out from the crowd due to their large single-ion magnetic anisotropy produced by the strong intrinsic spin-orbit coupling and the crystal-field effect [4-7]. However, most of the Ln-based SMMs with attractive high-performance are organometallic or air-sensitive complexes, the harsh synthetic conditions and intrinsic instability in air greatly restrict their future application [8-10].
On the other hand, switchable Ln-based SMMs via external stimuli, such as light, solvents, protonation, magnetic and electric fields, are of great importance and have attracted more attention in chemistry, physics and materials science [11-18]. Especially, light irradiation becomes an efficiently applied stimulus due to its convenient and rapid response for dynamic remote control and matched energy between different electronic states. A general approach is developed by incorporating photochromic moieties into the SMM system, including dithienylethene [19-21] with open and close forms, 1,2-bis(4pyridyl)ethene [22-26] and 9-anthrylmethylphosphonic acid [27] with photochemical cycloaddition, spiropyran-merocyanine $[28,29]$ with cis-trans isomerization and viologens [30,31] with photogenerated radicals.

In general, the slow magnetic relaxation in the spin crossover or metal-metal electron transfer complexes can be easily on/off switched via alternative light [32]. By comparison, SMM behaviors in the photochromic Ln-based SMMs could only be slightly tuned in the effective energy barrier and magnetic relaxation time. Actually, the construction of room-temperature light switchable on/off SMMs is still challenging in electron transfer photochromic materials. Recently, a Dy-based photochromic complex with photogenerated radical-actuated SMMs was realized in our previous work [30], showing the first example of SMMs in electron transfer photochromic materials. This motivates us to further explore the possibility of tunable SMMs via electron transfer photochromic complexes [32-38]. Especially, it is a challenging but interesting issue for switching off SMMs in these photochromic system. Furthermore, the operating temperature of light switch in these photochromic materials can be improved to room temperature, which is helpful for practical application.

Considering the above thoughts, two photochromic Ln-based complexes $\left[\mathrm{Ln}_{3}(\mathrm{H}-\mathrm{HEDP})_{2}\left(\mathrm{H}_{2}-\mathrm{HEDP}\right)_{2}\left(\mathrm{H}_{3}-\mathrm{HEDP}\right)_{2}\right] \cdot \mathrm{H}_{3}-\mathrm{TPP}$. $11 \mathrm{H}_{2} \mathrm{O}(\mathrm{Ln}=\mathrm{Dy}(\mathbf{1}), \mathrm{Tb}(2) ; \mathrm{TPP}=2,4,6$-tris(4-pyridyl)pyridine; HEDP = hydroxyethylidene diphosphonate) with changeable photomagnetic properties were reported. In these structures, electron-rich HEDP components served as electron donors, while electron-deficient $\mathrm{H}_{3}$-TPP motifs acted as acceptors; electron transfer process could occur after light irradiation via $\mathrm{H}$-bonding interactions. As a result, remarkable photochromic behavior was reversibly observed in both compounds, accompanied with the photogeneration of HEDP' and $\mathrm{H}_{3}$-TPP ${ }^{\bullet}$ radi-

College of Chemistry and Chemical Engineering, Qingdao University, Qingdao 266071, China

$\dagger$ These authors contributed equally to this work.

* Corresponding authors (emails: hujixiang@qdu.edu.cn (Hu JX); gmwang_pub@163.com (Wang GM)) 
cals. The magnetic behavior in these complexes was studied with characteristic single-molecule magnetic behavior. Importantly, originated from the antiferromagnetic couplings between the photogenerated radicals and $\mathrm{Ln}^{3+}$ ions, the SMM behavior in both compounds could be switched off after room-temperature light irradiation, showing the first example of radical-quenched SMM in molecule-based magnets. Furthermore, the SMM behavior in both compounds could be recovered to the initial state after eliminating photogenerated radicals via annealing treatment or pitting the colored samples in dark, providing a visual way for photo-swithchable SMM performance.

\section{EXPERIMENTAL SECTION}

\section{Materials and methods}

All the reagents and solvents for the syntheses of complexes were purchased from commercial sources and used as received. TPP ligand was purchased from Chemsoom and recrystallized in methanol solvent. Fourier transform infra-red (FT-IR) spectra were recorded in the range of $4000-400 \mathrm{~cm}^{-1}$ on a MAGNA-560 (Nicolet) FT-IR spectrometer (Canada). Powder X-ray diffraction (PXRD) patterns were collected with a Rigaku standard MiniFlex600 diffractometer (Japan). Ultraviolet-visible (UV-vis) spectra were measured using a Puxi Tu-1901 spectrophotometer (China). Elemental analyses for $\mathrm{C}, \mathrm{H}$ and $\mathrm{N}$ were determined on a PerkinElmer 240C analyzer (America). The fluorescence measurements were carried out on an F-4700 fluorescence spectrometer. Electron paramagnetic resonance (EPR) spectra were respectively collected at 298 and $100 \mathrm{~K}$ using a Bruker E500 EPR spectrometer (Germany). Magnetic measurements were obtained with the usage of a Quantum Design MPMS3 superconducting quantum interference device (SQUID) magnetometer (America).

Synthesis of $\left[\mathrm{Dy}_{3}(\mathrm{H}-\mathrm{HEDP})_{2}\left(\mathrm{H}_{2}-\mathrm{HEDP}\right)_{2}\left(\mathrm{H}_{3}-\mathrm{HEDP}\right)_{2}\right]$. $\mathrm{H}_{3}$-TPP.11H $\mathbf{O}$ (1)

$\mathrm{Dy}_{2} \mathrm{O}_{3}(0.091 \mathrm{~g}, 0.244 \mathrm{mmol})$, HEDP (0.207 g, $\left.0.924 \mathrm{mmol}\right), \mathrm{LiF}$ $(0.062 \mathrm{~g}, 2.08 \mathrm{mmol}$, acting as a mineralizer $)$, TPP $(0.037 \mathrm{~g}$, $0.119 \mathrm{mmol}$ ) were dissolved in the mixed solvents of methanol/ water $(3 \mathrm{~mL} / 2 \mathrm{~mL})$ in a $25-\mathrm{mL}$ Teflon-lined stainless steel vessel and heated at $120^{\circ} \mathrm{C}$ for $168 \mathrm{~h}$. The crystals were obtained by slowly cooling to $30^{\circ} \mathrm{C}$ in $12 \mathrm{~h}$. Yield: ca. $30 \%$ based on TPP ligands. 1a was obtained by Xe-lamp irradiation of compound $\mathbf{1}$ at room temperature for $40 \mathrm{~min}$. Elemental analysis for 1 (\%): calcd for $\mathrm{C}_{52} \mathrm{H}_{92} \mathrm{~N}_{8} \mathrm{O}_{53} \mathrm{P}_{12} \mathrm{Dy}_{3}$ (2536.47): C, 24.62; $\mathrm{H}, 3.65 ; \mathrm{N}$, 4.42. Found: C, 24.55; H, 3.73; N, 4.29. IR of 1 (KBr pellets, $\left.\mathrm{cm}^{-1}\right)$ : 3244(m), 3099(s), 2935(m), 2366(w), 2145(w), 1635(m), 1506(w), 1409(w), 1148(s), 1079(s), 925(m), 814(m), 656(w), 563(s), 463(w), 424(w). IR of 1a (KBr pellets, $\left.\mathrm{cm}^{-1}\right)$ : 3247(m), 3099(s), 2938(m), 2363(w), 2145(w), 1635(m), 1505(w), 1406(w), 1150(s), 1081(s), $922(\mathrm{~m}), 814(\mathrm{~m}), 654(\mathrm{w})$, 563(s), 462(w), 423(w).

Synthesis of $\left[\mathrm{Tb}_{3}(\mathrm{H}-\mathrm{HEDP})_{2}\left(\mathrm{H}_{2}-\mathrm{HEDP}\right)_{2}\left(\mathrm{H}_{3}-\mathrm{HEDP}\right)_{2}\right]$. $\mathrm{H}_{3}$-TPP.11H $\mathbf{O}$ (2)

$\mathrm{Tb}_{4} \mathrm{O}_{7}(0.089 \mathrm{~g}, 0.119 \mathrm{mmol})$, HEDP (0.189 g, $\left.0.843 \mathrm{mmol}\right), \mathrm{LiF}$ $(0.054 \mathrm{~g}, 2.08 \mathrm{mmol})$, TPP $(0.041 \mathrm{~g}, 0.133 \mathrm{mmol})$ were dissolved in the mixed solvents of methanol/water $(3 \mathrm{~mL} / 2 \mathrm{~mL})$ in a $25-\mathrm{mL}$ Teflon-lined stainless steel vessel and heated at $120^{\circ} \mathrm{C}$ for $168 \mathrm{~h}$. The crystals were obtained by slowly cooling to $30^{\circ} \mathrm{C}$ in 12 h. Yield: ca. 33\% based on TPP ligands. 2a was obtained by
Xe-lamp irradiation of compound 2 at room temperature for 40 min. Elemental analysis for $2(\%)$ : calcd for $\mathrm{C}_{52} \mathrm{H}_{92} \mathrm{~N}_{8} \mathrm{O}_{53} \mathrm{P}_{12}-$ $\mathrm{Tb}_{3}$ (2525.73): C, 24.73; H, 3.67; N, 4.43. Found: C, 24.60; $\mathrm{H}, 3.85 ; \mathrm{N}, 4.33$. IR of $2\left(\mathrm{KBr}\right.$ pellets, $\left.\mathrm{cm}^{-1}\right): 3424(\mathrm{~s}), 3252(\mathrm{~m})$, 3101(m), 2921(m), 2851(m), 2363(w), 2158(w), 1635(m), 1505(w), 1411(w), 1148(s), 1082(s), 923(w), 814(w), 654(w), $564(\mathrm{~m}), 464(\mathrm{w}), 421(\mathrm{w})$. IR of $2 \mathrm{a}\left(\mathrm{KBr}\right.$ pellets, $\left.\mathrm{cm}^{-1}\right): 3426(\mathrm{~s})$, 3248(m), 3100(m), 2919(m), 2851(m), 2363(w), 2158(w), 1632(m), 1507(w), 1411(w), 1148(s), 1082(s), 922(w), 814(w), 654(w), 563(m), 465(w), 421(w).

\section{RESULTS AND DISCUSSION}

\section{Description of crystal structures}

The single-crystal XRD analyses revealed that complexes $\mathbf{1}$ and 2 were isomorphic and belong to the orthorhombic crystal system with the space group $C 222_{1}$ (Table S1). Due to their same structures, complex 1 was discussed for clarity. The asymmetric unit was composed of one and a half $\mathrm{Dy}^{3+}$ ions, one protonated TPP ligand, three coordinated diphosphonate units, and several solvent water molecules (Fig. 1a). The distances of Dy-O were in the range of 2.224(6)-2.487(7) $\AA$ (Table S2), which was similar to those of previously reported $\mathrm{Dy}^{3+}$ complexes based on diphosphonate ligands [30]. The neighboring two $\mathrm{Dy}^{3+}$ ions were connected through diphosphonate groups to form a linear shaped chain (Fig. 1b). The closest intra-chain and inter-chain Dy...Dy distances were 4.8114(4) and 11.4279(9) $\AA$, respectively, indicating the dominant intra-chain interactions. Abundant $\mathrm{H}$ bonding interactions were formed between $\mathrm{N}$ atoms in the TPP ligand and the coordinated diphosphonate $\mathrm{O}$ atoms (Fig. $\mathrm{S} 1$ and Table S3), with an average H-bonding distance of $2.050 \AA$. These hydrogen bonds linked the chains to form a three-dimensional (3D) supramolecular framework (Fig. 1c). Furthermore, the strong $\mathrm{H}$-bonding interactions connected the HEDP electron donor and TPP acceptor, contributing to the fast electron transfer photochromism upon light irradiation. The geometrical configurations for $\mathrm{Ln}^{3+}$ ions were analyzed using SHAPE 2.0. As shown in Fig. 2, the Dy1 center was eight-coordinated by all $\mathrm{O}$ atoms from four distinct diphosphonate ligands, whereas Dy2 center adopted a hepta-coordination mode with the chelating phosphonate oxygen atoms, displaying a square antiprism and capped trigonal prism geometry, respectively (Tables S4 and S5), which was helpful for constructing molecular magnets with slow magnetic relaxation. The bond lengths and angles of 2 were similar with 1 and shown in Tables S6-S9.

\section{Photochromic properties}

The photochromic behavior of pure TPP ligand was firstly explored at solid state due to the large $\pi$-conjugated property and electron acceptability. After Xe-lamp light illumination, the color of TPP powders turned to faint yellow (Fig. S2, inset), showing the characteristics of the photochromism. The solidstate UV-vis and photoluminescence spectra were recorded to quest the light-induced color variations, with an appearance of a broad peak in UV-vis spectra and a decrease in emission spectra after irradiation, suggesting the generation of TPP radicals (Figs S2 and S3). Therefore, complexes 1 and $\mathbf{2}$ containing TPP ligands may display photochromic behavior due to photoinduced electron transfer from electron-rich groups to TPP ligand via hydrogen-bonding interactions. As expected, both complexes were very sensitive to light, and naked-eye-detectable 
a

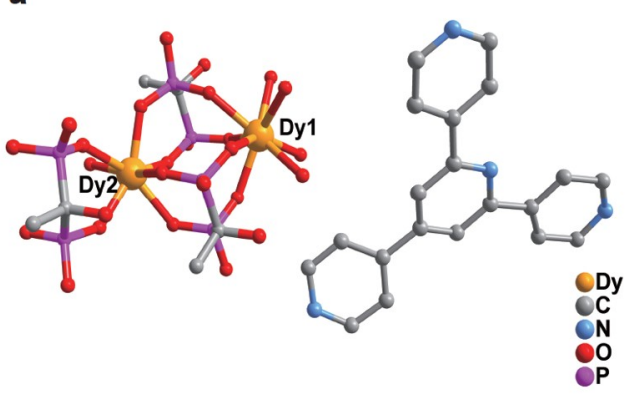

b

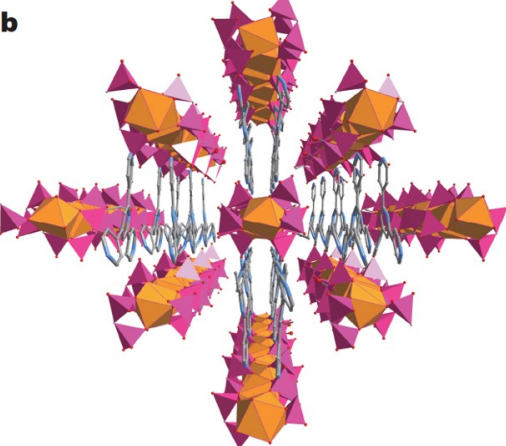

c

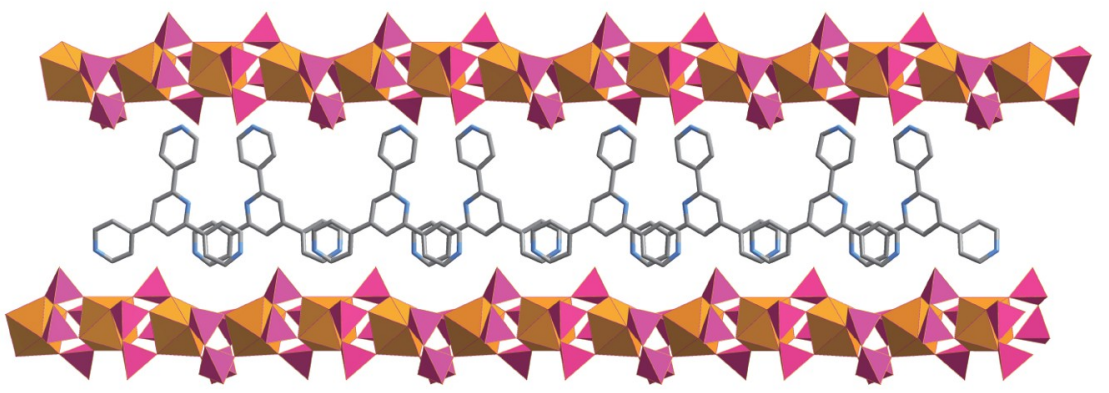

Figure 1 (a) Asymmetric unit for compound 1. For clarity, water and $\mathrm{H}$ atoms were omitted; (b) 1D linear shaped chain for compound 1; (c) 3D supramolecular structure of compound 1. Atom legend: P, pink; N, blue; O, red; C, gray-40\%; Dy, light orange.
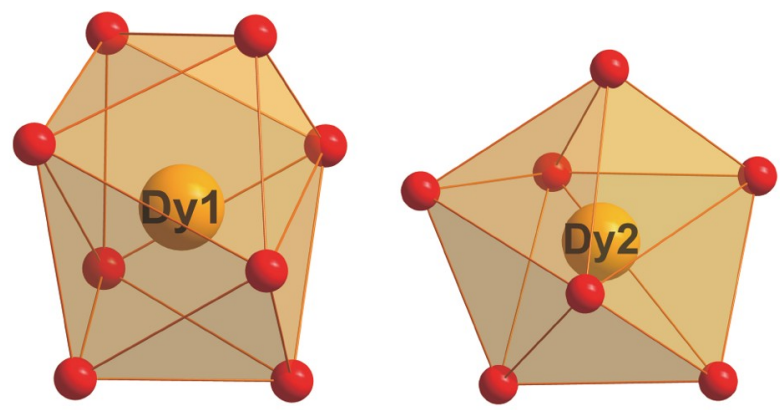

Figure 2 Coordination environments of $\mathrm{Dy}^{3+}$ ions in compound $\mathbf{1}$.

and rapid color changes from yellow to purple were observed upon exposure to Xe-lamp for $1 \mathrm{~min}$ at room temperature (Fig. 3, inset). The photosensitive products could be decolorized via putting photoactivated samples in dark for three days or heating the samples at $140^{\circ} \mathrm{C}$ for $5 \mathrm{~h}$, showing a completely reversible photochromism. The reversibility of coloration and decoloration process for both compounds was also performed using the in-situ fluorescence spectra, which could be cycled for at least five times (Fig. S4). The unchanged PXRD and IR patterns in both compounds during reversible photochromism indicated that the photochromism was not caused by structural transformation or photolysis (Figs S5-S7). The solid-state timedependent UV-vis spectra showed that the photoproducts of 1 and 2 generated new absorption bands around $570 \mathrm{~nm}$, which was ascribed to the photogeneration of $\mathrm{H}_{3}$-TPP free radicals (Fig. 3a, b). The intensities of the peaks gradually increased with the prolonged irradiation time, showing a radical-induced photochromic process. After decoloration, this $\mathrm{H}_{3}$-TPP radical peak disappeared and the curve returned to the as-prepared state, showing a reversible photochromic process. By contrast, the HEDP electron donor lost electrons and formed the HEDP* radicals via migrating electrons to $\mathrm{H}_{3}$-TPP units, providing additional spin carriers to magnetically couple with $\mathrm{Ln}^{3+}$ ions and tune SMM behavior.

To confirm the generation of radicals, the solid-state electron spin resonance (ESR) spectra of both complexes before and after irradiation were measured. As shown in Fig. 3c, d, the original samples 1 and 2 revealed the signal of the rare-earth metal ions between 500 and $6500 \mathrm{G}$, and the weak signals may be due to the fast spin-lattice relaxation. After light irradiation, sharp signals centered at $g=2.014$ and 1.996 occurred for 1a and 2a, characteristic of the free radicals (2.0023), and the small deviations should originate from the couplings between $\mathrm{Ln}^{3+}$ ions and photogenerated radicals. Therefore, the photochromic behaviors of both complexes should be stemmed from the photogeneration of radicals via ligand-to-ligand electron transfer process. To indepth explore the variations of magnetic couplings between $\mathrm{Ln}^{3+}$ ions under the presence of photogenerated radicals, low-temperature EPR spectra of $\mathbf{1}$ and $\mathbf{2}$ were collected at a frequency of $9.41 \mathrm{GHz}$ at $100 \mathrm{~K}$ before and after light irradiation, in which weak exchanges could be better defined even though the fast spin-lattice relaxation of the $\mathrm{Ln}^{3+}$ ions. As shown in Figs S8-S11, the signals assigned to $\mathrm{Ln}^{3+}$ ions largely enhanced after irradiation, and the radical...Ln exchange remarkably perturbed the EPR spectra of both compounds upon lowing the temperature, further indicating magnetic couplings between photogenerated HEDP radicals and $\mathrm{Ln}^{3+}$ ions. The decreased intensity in radical signal also implied the singlet diradicals in these structures [39]. Furthermore, room- and low-temperature EPR spectra also suggested anisotropy of $g$ factor, which made it possible to treat these compounds as Ising systems at low temperatures [40].

The solid-state luminescence spectra for both compounds were also studied at room temperature. As depicted in Fig. 4a, the emission maximum was obtained at $376 \mathrm{~nm}$ for $\mathbf{1}\left(\lambda_{\mathrm{ex}}=\right.$ 

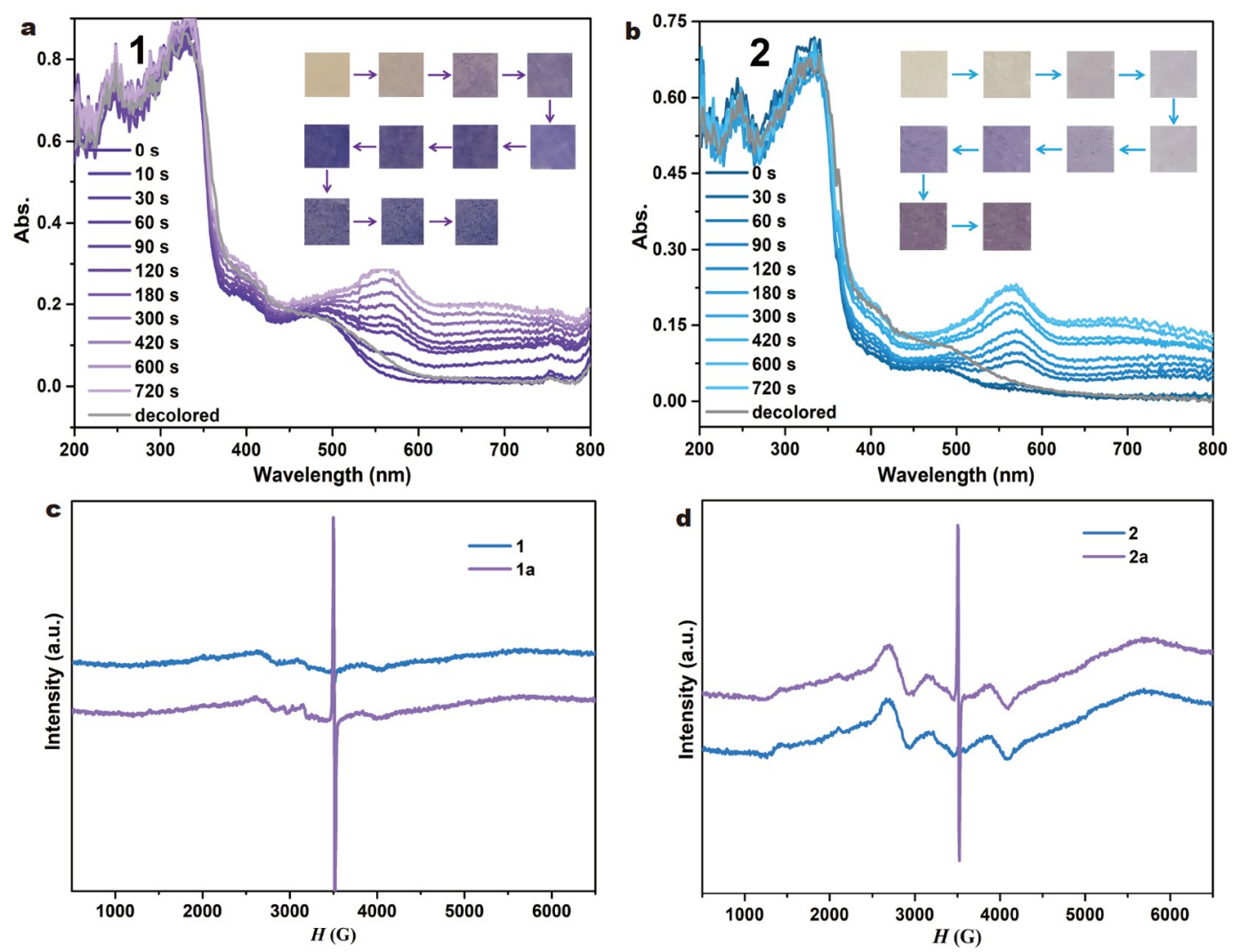

Figure 3 Time-dependent UV-vis spectra of 1 (a) and $\mathbf{2}$ (b). Insets: color changes during UV-vis spectral measurements. Room-temperature ESR spectra of $\mathbf{1}$ and $\mathbf{1 a}(\mathrm{c}), \mathbf{2}$ and $\mathbf{2 a}(\mathrm{d})$ at solid state.
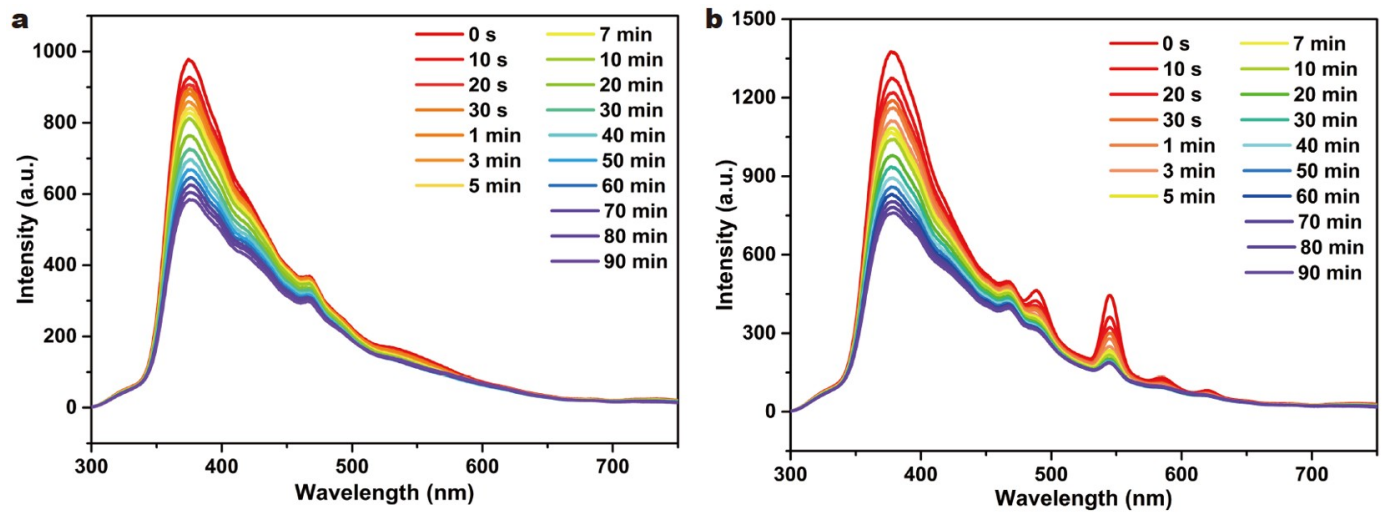

Figure 4 The variation of emission spectra of 1 (a) and $\mathbf{2}$ (b) at different irradiation time points.

$270 \mathrm{~nm}$ ), which was attributed to the $\pi^{*}-\pi$ transition in TPP ligand. Moreover, the $4 \mathrm{~F}_{9 / 2} \rightarrow 6 \mathrm{H}_{J}(J=15 / 2)$ transition of $\mathrm{Dy}^{3+}$ ion caused a small emission peak at $467 \mathrm{~nm}$. Similar with 1, the strongest emission peak of 2 was located at $376 \mathrm{~nm}$, which was also corresponded to the transition of ligand. The emission spectrum of 2 (Fig. $4 \mathrm{~b}$ ) consisted of the main bands at 488.8 , $544.8,585,619.6 \mathrm{~nm}$, such emission originated from the $\mathrm{f}-\mathrm{f}$ transition lines within the $4 \mathrm{f}^{8}$ electron configuration of $\mathrm{Tb}^{3+}$, assigned to ${ }^{5} \mathrm{D}_{4} \rightarrow{ }^{7} \mathrm{~F}_{J}(J=6-3)$ transitions of the $\mathrm{Tb}^{3+}$ ion $[41$ -44]. Interestingly, with the extension of irradiation time, the intensities in both compounds decreased gradually, and finally unchanged after 90-min irradiation. Since the absorption of $\mathrm{H}_{3^{-}}$ TPP radicals covered the emission of $\mathrm{Dy}^{3+}$ and $\mathrm{Tb}^{3+}$ ions, the characteristic emission of $\mathrm{Ln}^{3+}$ ions after irradiation showed a decreasing trend, resulting in the reduction of all fluorescence emission peaks. This indicated that both 1 and 2 possessed photomodulated fluorescence behaviors. The quenched phenomenon could be attributed to the intermolecular energy transfer from the luminescent excited $\mathrm{Ln}^{3+}$ centers to the photogenerated radicals.

\section{Magnetic studies}

Due to the anisotropy of $\mathrm{Dy}^{3+}$ and $\mathrm{Tb}^{3+}$ ions, the magnetic measurements were performed to explore the switch of SMM behavior via photogenerated radicals. The direct current (DC) magnetic susceptibility under a DC field of 1000 Oe for both compounds (Fig. 5) showed that, the $\chi_{\mathrm{M}} T$ value $\left(\chi_{\mathrm{M}}\right.$ is the molar susceptibility and $T$ is the temperature) at $300 \mathrm{~K}$ was 42.62 and 


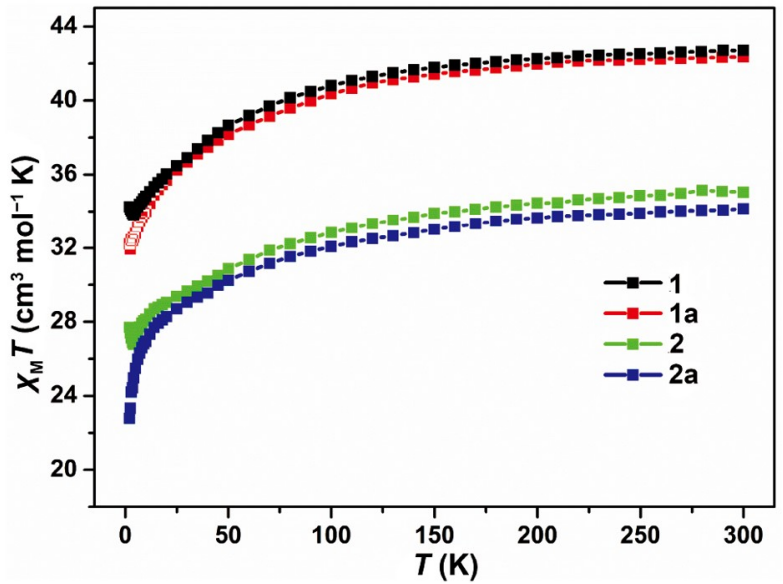

Figure 5 Temperature-dependent of $\chi_{\mathrm{M}} T$ products for 1 and 2 before and after light irradiation under a $1000-O e$ DC field.

$35.21 \mathrm{~cm}^{3} \mathrm{~K} \mathrm{~mol}^{-1}$ for $\mathbf{1}$ and $\mathbf{2}$, respectively, which was close to the expected value for three uncoupled $\mathrm{Dy}^{3+}\left(42.51 \mathrm{~cm}^{3} \mathrm{~mol}^{-1} \mathrm{~K}\right.$, $S=5 / 2, L=5, g=4 / 3)$ and $\mathrm{Tb}^{3+}\left(35.46 \mathrm{~cm}^{3} \mathrm{~mol}^{-1} \mathrm{~K}, S=3, L=3\right.$, $g=3 / 2)$ ions. As the temperature decreased, the $\chi_{\mathrm{M}} T$ values decreased gradually and reached a minimum value of 33.8 and $26.8 \mathrm{~cm}^{3} \mathrm{~K} \mathrm{~mol}^{-1}$ at about $4 \mathrm{~K}$, which was attributed to the decrease of $m_{J}$ sublevels and commonly seen in other Ln-based complexes. Then the $\chi_{\mathrm{M}} T$ values slightly increased, suggesting weak ferromagnetic couplings between the intrachain $\mathrm{Ln}^{3+}$ centers. After being irradiated with a Xe-lamp at room temperature for about $40 \mathrm{~min}$, large radicals via photogeneration could induce magnetic couplings with $\mathrm{Ln}^{3+}$ centers. As shown in Fig. 5, the $\chi_{M} T$ values at room temperature decreased to 41.79 and $33.33 \mathrm{~cm}^{3} \mathrm{~K} \mathrm{~mol}^{-1}$ for $\mathbf{1}$ and $\mathbf{2}$. Apparently, the decreases in light-response magnetization were due to the antiferromagnetic couplings between $\mathrm{Ln}^{3+}$ ions and photogenerated stable radicals, similar to other electron-transfer photochromic materials [33]. Then $\chi_{M} T-T$ curves directly decreased at low temperatures, dif- ferent from the curves of the as-prepared samples. The magnetization values for both compounds in the $M-H$ curves ( $M$ is the magnetization and $H$ is the magnetic field) at $2 \mathrm{~K}$ exhibited obvious decreases at high fields after light irradiation, indicating the appearance of antiferromagnetic exchange interactions between these spin carries (Figs S12 and S13).

To comprehensively understand the effect of photogenerated radicals on dynamic magnetic properties, the temperature dependence of alternating current (AC) magnetic susceptibilities were measured on microcrystalline samples for both compounds. For 1, both the in-phase $\left(\chi^{\prime}\right)$ and out-of-phase $\left(\chi^{\prime \prime}\right)$ components in the frequency-dependent AC susceptibility data exhibited frequency dependence under a zero DC and 3.5-Oe AC field (Fig. S14), showing the slow magnetic relaxation behavior. However, originated from fast quantum tunnelling of magnetization (QTM), no peaks for both $\chi^{\prime}$ and $\chi^{\prime \prime}$ components were observed even at $2 \mathrm{~K}$. To restrain the rapid relaxation of magnetization, a DC magnetic field of 2000 Oe was applied [45] and the $\chi^{\prime}$ and $\chi^{\prime \prime}$ components showed more obvious frequency dependence (Fig. 6a), indicating the presence of magnetic relaxation and field-induced single-molecule magnetic behavior. The Debye model based on $\ln \left(\chi^{\prime \prime} / \chi^{\prime}\right)=\ln \left(\omega \tau_{0}\right)+E_{\mathrm{a}} / k_{\mathrm{B}} T$ was also utilized to estimate the energy barrier $E_{\mathrm{a}}$ and preexponential factor $\tau_{0}$, where $\omega$ is the frequency and $k_{\mathrm{B}}$ is the Boltzmann constant. A linear fitting result yielded $E_{\mathrm{a}} / k_{\mathrm{B}}=16.9 \mathrm{~K}$ and $\tau_{0}=$ $8.4 \times 10^{-6} \mathrm{~s}$ (Fig. S15), consistent with a superparamagnetic-like character of the relaxation dynamics and confirming the SMM behavior [46,47]. Interestingly, the irradiated samples of 1a showed a different dynamic magnetic behavior from that of complex 1 . The frequency dependence in both $\chi^{\prime}$ and $\chi^{\prime \prime}$ components nearly disappeared after light irradiation even in an applied 2000-Oe DC field (Fig. S16), showing a photogenerated radical-quenched SMM behavior. After decoloration, both the $\chi^{\prime}$ and $\chi^{\prime \prime}$ components in the AC data returned to the initial state of the as-prepared samples of $\mathbf{1}$ (Fig. S17), showing the switchable off/on SMMs via light and heat treatment. For 2, both the $\chi^{\prime}$ and $\chi^{\prime \prime}$ components (Fig. 6b) showed frequency dependence under a
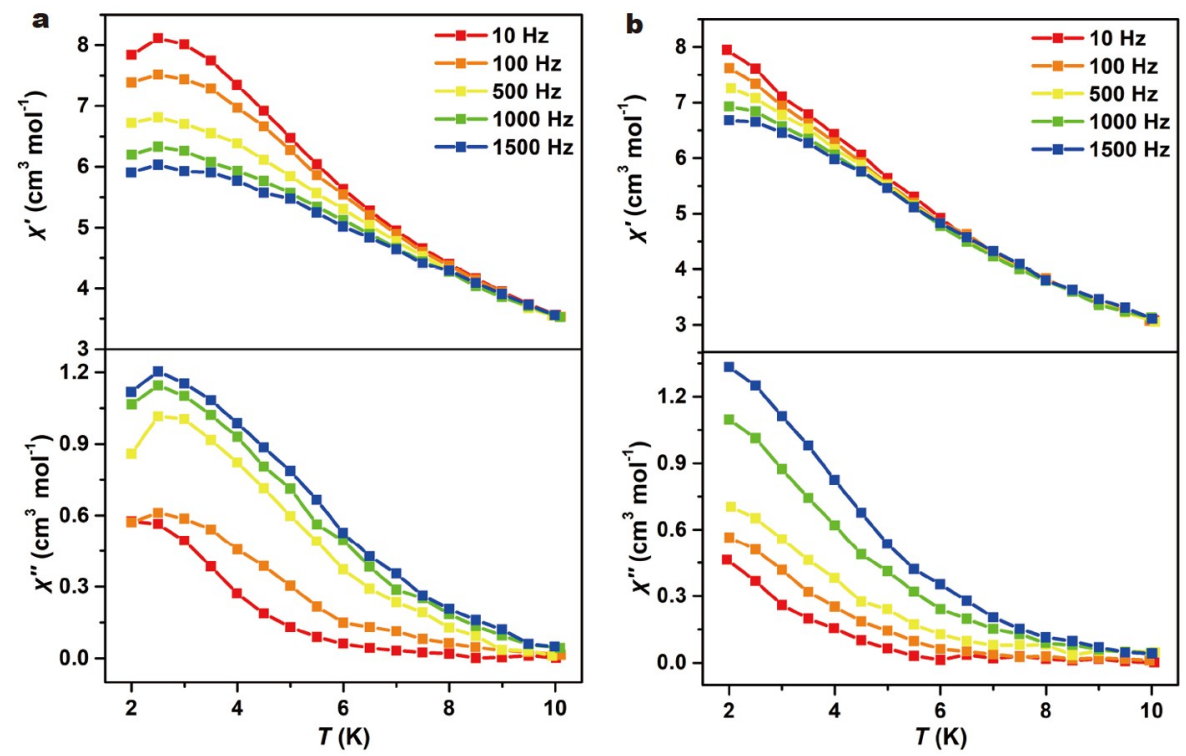

Figure 6 Temperature dependence of the in-phase $\left(\chi^{\prime}\right)$ and out-of-phase $\left(\chi^{\prime \prime}\right)$ AC susceptibilities for $\mathbf{1}(\mathrm{a})$ and 2 (b) measured at different frequencies in a 3.5-Oe AC and 2000-Oe DC field. 
2000-Oe DC field, with $E_{\mathrm{a}} / k_{\mathrm{B}}=12.1 \mathrm{~K}$ and $\tau_{0}=8.1 \times 10^{-6} \mathrm{~s}$ (Fig. S18). After light irradiation, the photogenerated HEDP* radicals antiferromagnetically coupled with $\mathrm{Tb}^{3+}$ ions, inducing the total disappearance of slow magnetic relaxations, even measured the AC data with a 2000-Oe DC field (Fig. S19).

\section{CONCLUSIONS}

In summary, two Ln-based chain complexes were synthesized and their structures, photochromic behavior and photomagnetic properties were investigated. The room-temperature reversible photo-coloration phenomenon was induced by photogenerated radicals with an electron transfer process from electron-rich diphosphonate units to electron-deficient $\mathrm{H}_{3}$-TPP components, characterized by structural analyses, UV-vis, luminescence and ESR spectra and magnetic measurements. Both complexes showed obviously field-induced SMM behavior before light irradiation. Interestingly, accompanied with coloration, the SMM behavior could be switched off/on through light irradiation and heat treatment, for the first time realizing the off SMM behavior by photogenerated radicals in the molecule-based magnets. Based on electron transfer-triggered photochromism, this work constructs a system for switchable SMMs, providing a visual operation way through eye-detectable coloration for the on-off switchable SMMs. More studies on the photogenerated radicals switched SMMs are on the way.

Received 1 October 2021; accepted 9 November 2021; published online 15 December 2021

1 Sessoli R, Tsai HL, Schake AR, et al. High-spin molecules: [ $\mathrm{Mn}_{12} \mathrm{O}_{12}{ }^{-}$ $\left.\left(\mathrm{O}_{2} \mathrm{CR}\right)_{16}\left(\mathrm{H}_{2} \mathrm{O}\right)_{4}\right]$. J Am Chem Soc, 1993, 115: 1804-1816

2 Meng YS, Liu T. Manipulating spin transition to achieve switchable multifunctions. Acc Chem Res, 2019, 52: 1369-1379

3 Wu DQ, Shao D, Wei XQ, et al. Reversible on-off switching of a singlemolecule magnet via a crystal-to-crystal chemical transformation. J Am Chem Soc, 2017, 139: 11714-11717

4 Zhu Z, Zhao C, Feng T, et al. Air-stable chiral single-molecule magnets with record anisotropy barrier exceeding $1800 \mathrm{~K}$. J Am Chem Soc, 2021, 143: 10077-10082

5 Liu K, Zhang X, Meng X, et al. Constraining the coordination geometries of lanthanide centers and magnetic building blocks in frameworks: A new strategy for molecular nanomagnets. Chem Soc Rev, 2016, 45: 2423-2439

6 Liu JL, Chen YC, Tong ML. Symmetry strategies for high performance lanthanide-based single-molecule magnets. Chem Soc Rev, 2018, 47: 2431-2453

7 Woodruff DN, Winpenny REP, Layfield RA. Lanthanide single-molecule magnets. Chem Rev, 2013, 113: 5110-5148

8 Rinehart JD, Fang M, Evans WJ, et al. $\mathrm{A} \mathrm{N}_{2}{ }^{3-}$ radical-bridged terbium complex exhibiting magnetic hysteresis at 14 K. J Am Chem Soc, 2011, 133: $14236-14239$

9 Blagg RJ, Ungur L, Tuna F, et al. Magnetic relaxation pathways in lanthanide single-molecule magnets. Nat Chem, 2013, 5: 673-678

10 Liu J, Chen YC, Liu JL, et al. A stable pentagonal bipyramidal Dy(III) single-ion magnet with a record magnetization reversal barrier over 1000 K. J Am Chem Soc, 2016, 138: 5441-5450

11 Sato O. Dynamic molecular crystals with switchable physical properties. Nat Chem, 2016, 8: 644-656

12 Sato O, Tao J, Zhang YZ. Control of magnetic properties through external stimuli. Angew Chem Int Ed, 2007, 46: 2152-2187

13 Komeda $\mathrm{T}$, Isshiki $\mathrm{H}$, Liu J, et al. Observation and electric current control of a local spin in a single-molecule magnet. Nat Commun, 2011, 2: 217

14 Zhou Q, Yang F, Xin B, et al. Reversible switching of slow magnetic relaxation in a classic lanthanide metal-organic framework system. Chem Commun, 2013, 49: 8244-8246
15 Liu JL, Chen YC, Zheng YZ, et al. Switching the anisotropy barrier of a single-ion magnet by symmetry change from quasi- $D_{5 \mathrm{~h}}$ to quasi- $O_{\mathrm{h}}$. Chem Sci, 2013, 4: 3310-3316

16 Feng X, Mathonière C, Jeon IR, et al. Tristability in a light-actuated single-molecule magnet. J Am Chem Soc, 2013, 135: 15880-15884

17 Suzuki K, Sato R, Mizuno N. Reversible switching of single-molecule magnet behaviors by transformation of dinuclear dysprosium cores in polyoxometalates. Chem Sci, 2013, 4: 596-600

18 Liang Z, Damjanović M, Kamila M, et al. Proton control of the lanthanoid single-ion magnet behavior of a double-decker complex with an indolenine-substituted annulene ligand. Inorg Chem, 2017, 56: 6512-6521

19 Irie M. Diarylethenes for memories and switches. Chem Rev, 2000, 100: 1685-1716

20 Morimoto M, Miyasaka H, Yamashita M, et al. Coordination assemblies of $\left[\mathrm{Mn}_{4}\right]$ single-molecule magnets linked by photochromic ligands: Photochemical control of the magnetic properties. J Am Chem Soc, 2009, 131: 9823-9835

21 Tian H, Yang S. Recent progresses on diarylethene based photochromic switches. Chem Soc Rev, 2004, 33: 85-97

22 Wang LF, Qiu JZ, Chen YC, et al. [2 + 2] Photochemical modulation of the Dy(III) single-molecule magnet: Opposite influence on the energy barrier and relaxation time. Inorg Chem Front, 2017, 4: 1311-1318

23 Ou YC, Liu WT, Li JY, et al. Solvochromic and photodimerization behaviour of $1 \mathrm{D}$ coordination polymer via single-crystal-to-singlecrystal transformation. Chem Commun, 2011, 47: 9384-9386

24 Nagarathinam M, Vittal JJ. Photochemical [2 +2] cycloaddition as a tool to study a solid-state structural transformation. Chem Commun, 2008, 8: 438-440

25 Park IH, Medishetty R, Kim JY, et al. Distortional supramolecular isomers of polyrotaxane coordination polymers: Photoreactivity and sensing of nitro compounds. Angew Chem Int Ed, 2014, 53: 5591-5595

26 Wang LF, Qiu JZ, Liu JL, et al. Modulation of single-molecule magnet behaviour via photochemical [2 +2$]$ cycloaddition. Chem Commun, 2015, 51: 15358-15361

27 Tian H, Su JB, Bao SS, et al. Reversible ON-OFF switching of singlemolecule-magnetism associated with single-crystal-to-single-crystal structural transformation of a decanuclear dysprosium phosphonate. Chem Sci, 2018, 9: 6424-6433

28 Paquette MM, Plaul D, Kurimoto A, et al. Opto-spintronics: Photoisomerization-induced spin state switching at $300 \mathrm{~K}$ in photochrome cobalt-dioxolene thin films. J Am Chem Soc, 2018, 140: 14990-15000

29 Zhang J, Zou Q, Tian H. Photochromic materials: More than meets the eye. Adv Mater, 2013, 25: 378-399

30 Ma YJ, Hu JX, Han SD, et al. Manipulating on/off single-molecule magnet behavior in a Dy(III)-based photochromic complex. J Am Chem Soc, 2020, 142: 2682-2689

31 Zhang Q, Wei WJ, Li Q, et al. Light enhanced proton conductivity in a terbium phosphonate photochromic chain complex. Sci China Chem, 2021, 64: 1170-1176

32 Zhao L, Meng YS, Liu Q, et al. Switching the magnetic hysteresis of an $\left[\mathrm{Fe}^{\mathrm{II}}-\mathrm{NC}-\mathrm{W}^{\mathrm{V}}\right]$-based coordination polymer by photoinduced reversible spin crossover. Nat Chem, 2021, 13: 698-704

33 Cai LZ, Chen QS, Zhang CJ, et al. Photochromism and photomagnetism of a $3 \mathrm{~d}-4 \mathrm{f}$ hexacyanoferrate at room temperature. J Am Chem Soc, 2015, 137: 10882-10885

34 Cai LZ, Guo PY, Wang MS, et al. Photoinduced magnetic phase transition and remarkable enhancement of magnetization for a photochromic single-molecule magnet. J Mater Chem C, 2021, 9: 22312235

35 Li Q, Zhang Q, Wei WJ, et al. Light actuated stable radicals of the 9anthracene carboxylic acid for designing new photochromic complexes. Chem Commun, 2021, 57: 4295-4298

36 Hu JX, Jiang XF, Ma YJ, et al. Optically actuating ultra-stable radicals in a large $\pi$-conjugated ligand constructed photochromic complex. Sci China Chem, 2021, 64: 432-438

37 Han SD, Liu AU, Wei Q, et al. Quadruple photoresponsive functionality in a crystalline hybrid material: Photochromism, photomodulated fluorescence, magnetism and nonlinear optical properties. Chem Eur J, 


\section{1, 27: 7842-7846}

38 Liu AJ, Xu F, Han SD, et al. Mixed-ligand strategy for the construction of photochromic metal-organic frameworks driven by electron-transfer between nonphotoactive units. Cryst Growth Des, 2020, 20: 7350-7355

39 Chandra Mondal K, Roy S, Roesky HW. Silicon based radicals, radical ions, diradicals and diradicaloids. Chem Soc Rev, 2016, 45: 1080-1111

40 Amjad A, Madalan AM, Andruh M, et al. Slow relaxation of magnetization in an isostructural series of zinc-lanthanide complexes: An integrated EPR and AC susceptibility study. Chem Eur J, 2016, 22: 12849-12858

41 Wang H, Liu SJ, Tian D, et al. Temperature-dependent structures of lanthanide metal-organic frameworks based on furan-2,5-dicarboxylate and oxalate. Cryst Growth Des, 2012, 12: 3263-3270

42 Wang HS, Zhao B, Zhai B, et al. Syntheses, structures, and photoluminescence of one-dimensional lanthanide coordination polymers with 2,4,6-pyridinetricarboxylic acid. Cryst Growth Des, 2007, 7: 18511857

43 Zhang SY, Liao JS, Xu WJ, et al. Structural phase transitions, dielectric bistability and luminescence of two bulky ion-pair crystals $\left[\mathrm{N}\left(\mathrm{C}_{3} \mathrm{H}_{7}\right)_{4}\right]_{2}-$ $\left[\mathrm{Ln}\left(\mathrm{NO}_{3}\right)_{5}\right](\mathrm{Ln}=\mathrm{Tb}$, Dy). New J Chem, 2017, 41: 9963-9968

44 Hou YL, Cheng RR, Xiong G, et al. Structures, luminescent and magnetic properties of a series of $(3,6)$-connected lanthanide-organic frameworks. Dalton Trans, 2014, 43: 1814-1820

45 Han SD, Miao XH, Liu SJ, et al. Magnetocaloric effect and slow magnetic relaxation in two dense $(3,12)$-connected lanthanide complexes. Inorg Chem Front, 2014, 1: 549-552

46 Li H, Sun J, Yang M, et al. Functionalized nitronyl nitroxide biradical bridged one-dimensional lanthanide chains: Slow magnetic relaxation in the $\mathrm{Tb}$ and Dy analogues. New J Chem, 2017, 41: 10181-10188

47 Guo YN, Xu GF, Gamez P, et al. Two-step relaxation in a linear tetranuclear dysprosium(III) aggregate showing single-molecule magnet behavior. J Am Chem Soc, 2010, 132: 8538-8539

Acknowledgements This work was supported by the National Natural Science Foundation of China (21901133, 22171155 and 22071126) and the State Key Laboratory of Fine Chemicals (KF 1905).

Author contributions Zhang Q, Han SD and Li Q performed the experiments; Hu JX, Zhang Q and Han SD performed the data analysis; Hu JX wrote the paper; Hu JX and Wang GM designed the concept and supervised the experiments. All authors contributed to the general discussion.

Conflict of interest The authors declare that they have no conflict of interest.

Supplementary information Supporting data are available in the online version of the paper. CCDC 2097403 and 2097404 for $\mathbf{1}$ and $\mathbf{2}$ contains the supplementary crystallographic data for this paper. These data can be obtained free of charge from The Cambridge Crystallographic Data Centre via www.ccdc.cam.ac.uk/data_request/cif.

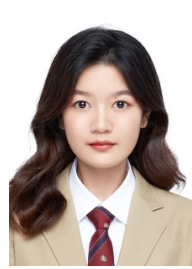

Qian Zhang was born in 1997 in Shandong, China. She obtained her BSc degree from Qingdao University in 2019. Now she is a master student under the supervision of Prof. Guo-Ming Wang. Her research is focused on the exploration of the photochromic properties of metal phosphonates.

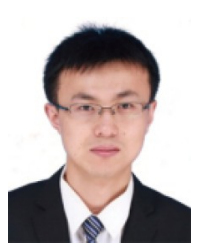

Song-De Han was born in 1987 in Shandong, China. He received his BSc degree from Qingdao University in 2010, and $\mathrm{PhD}$ degree (2015) in chemistry from Nankai University under the supervision of Prof. Xian-He Bu. In 2015, he joined the faculty of Qingdao University. He is currently an associate professor at the College of Chemistry and Chemical Engineering. His current research interests include the design and synthesis of crystalline hybrid materials with focus on photochromism, and room-temperature phosphorescence.

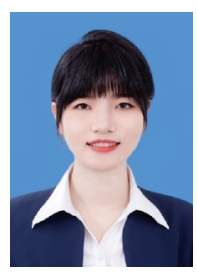

Qi Li was born in 1997 in Shandong, China. She obtained her BSc degree from Qingdao University in 2019. Now she is a master student under the supervision of Prof. Ji-Xiang $\mathrm{Hu}$. Her research focuses on the design and construction of photomagnetic systems.

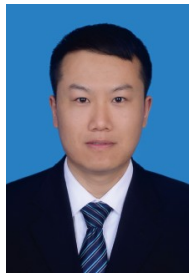

Ji-Xiang Hu was born in 1988 in Shandong, China. He earned his $\mathrm{PhD}$ degree (2018) in chemistry from Dalian University of Technology under the supervision of Prof. Tao Liu. In 2018, he joined Qingdao University and became a full professor. His research interests are related to the design and synthesis of photomagnetic molecules and spin crossover complexes, and the dynamic control of the hybrid materials.

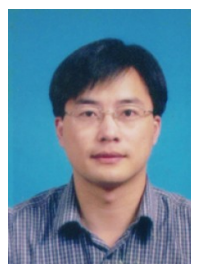

Guo-Ming Wang was born in 1977 in Shandong, China. He obtained his PhD degree from Fujian Institute of Research on the Structure of Matter, Chinese Academy of Sciences (CAS) in 2005 under the supervision of Prof. Guo-Yu Yang. In 2006, he joined Qingdao University and became a full professor in 2012. His current research interest focuses on the design and synthesis of crystalline framework materials with room-temperature phosphorescence, photochromism, photomagnetism and other photo-modulated physical properties.

\section{$\operatorname{Ln}$ 基 $(\operatorname{Ln}=\mathrm{Dy}, \mathrm{Tb})$ 配合物的电子转移光致变色及其 可逆开关单分子磁体行为}

张倩 ${ }^{\dagger}$, 韩松德 ${ }^{\dagger}$, 李琪, 胡继祥 ${ }^{*}$, 王国明*

摘要 室温下实现单分子磁体 $(\mathrm{SMM})$ 的开关行为仍然是分子磁体研究 中的一个挑战. 本文合成了两例具有 SMM行为的光致变色 $\operatorname{Ln}$ 基 $(\mathrm{Ln}=$ $\mathrm{Dy}, \mathrm{Tb})$ 膦酸盐配合物. 结构分析、紫外-可见光谱、发光光谱、电子 自旋共振光谱和磁性测量等手段共同表明, 目标产物的室温可逆光致 变色行为是光生自由基诱导的电子转移过程. 重要的是, 由于 $\mathrm{Ln}^{3+}$ 与光 生自由基间的反铁磁耦合作用, 首次观测到室温光照可猝灭SMM行为. 此外, 通过热处理消除光生自由基后, SMM的行为得以恢复, 因而通过 光照和加热实现了可逆的 SMM关/开变化. 本工作在电子转移光致变色 体系中实现 SMM的可控开/关, 为可调控的SMM提供了一种可视化的 操作方式. 